\title{
IDENTIFYING SEVERAL BIASED COINS ENCOUNTERED BY A HIDDEN RANDOM WALK
}

\author{
David A. Levin ${ }^{1}$ And Yuval Peres ${ }^{2}$
}

FEBRUARY 9, 2004

\begin{abstract}
Suppose that attached to each site $z \in \mathbb{Z}$ is a coin with bias $\theta(z)$, and only finitely many of these coins have non-zero bias. Allow a simple random walker to generate observations by tossing, at each move, the coin attached to its current position. Then we can determine the biases $\{\theta(z)\}_{z \in \mathbb{Z}}$, using only the outcomes of these coin tosses and no information about the path of the random walker, up to a shift and reflection of $\mathbb{Z}$. This generalizes a result of Harris and Keane.
\end{abstract}

KEYWORDS: random walks, reconstruction

Subject Classification: Primary: 60J15, Secondary: 62M05

\section{Introduction}

A coin toss with bias $\theta$ is a $\{-1,1\}$-valued random variable with expected value $\theta$, and a fair coin is a coin toss with bias zero.

Harris and Keane [HK97] introduced a model for sequences of coin tosses with a particular kind of dependence. Let $S=\left\{S_{n}\right\}_{n=0}^{\infty}$ be simple random walk on $\mathbb{Z}$. Suppose that whenever $S_{n}=0$, an independent coin with bias $\theta \geq 0$ is tossed, while at all other times an independent fair coin is tossed. Suppose that we are given $X=\left\{X_{n}\right\}_{n=0}^{\infty}$, the record of coin tosses obtained, but the path of the walker, $S$, is hidden from us. Can $X$ be distinguished from a sequence of i.i.d. fair coin tosses?

If it can be distinguished, can the parameter $\theta$ be recovered, again only from $X$ ?

Harris and Keane proved the following:

Theorem ([HK97, Theorem 2]). There exist a sequence of functions $\Theta_{n}:\{-1,1\}^{n} \rightarrow \mathbb{R}$, not depending on $\theta$, so that $\lim _{n \rightarrow \infty} \Theta_{n}\left(X_{1}, \ldots, X_{n}\right)=\theta$ almost surely.

\footnotetext{
${ }^{1}$ Department of Mathematics, University of Utah, Salt Lake City, UT 84112. levin@math.utah.edu.

${ }^{2}$ Departments of Statistics and Mathematics, University of California, Berkeley, CA 94720. peres@stat.berkeley.edu. http://stat-www.berkeley.edu/ peres. Research supported in part by NSF Grants DMS-0104073 and DMS-0244479.
} 
In fact, the model in [HK97] is more general, allowing the walk to be any null-recurrent Markov chain. Let $\mu_{\theta}$ be the distribution of $X$ on $\{-1,1\}^{\mathbb{N}}$. Recall that two Borel measures are mutually singular if there is a Borel set which is null for one measure such that its complement is null for the other measure; in this case the measures are distinguished by almost any observation. Whether or not $\mu_{\theta}$ and $\mu_{0}$ are mutually singular depends on the graph, and as was shown in [LPP01], sometimes on the bias $\theta$. [HK97] provides a partial criterion for determining singularity based on the graph, while the full story - in particular the role of $\theta$ for certain graphs - is completed in [LPP01].

In this paper, this model is generalized in a different direction. Label each $z \in \mathbb{Z}$ with $\theta(z) \in[0,1]$. Now allow a random walker to move on $\mathbb{Z}$, and suppose that on her $n$th move she is at position $S_{n}$ and she tosses a coin with bias $\theta\left(S_{n}\right)$, generating the random variable $X_{n}$. Again, the path of the walker, $\left\{S_{n}\right\}_{n=0}^{\infty}$ is not observed, and the output is only the outcomes of the coin tosses, that is, $\left\{X_{n}\right\}_{n=0}^{\infty}$. The main result of this paper says that the biases $\{\theta(z): z \in \mathbb{Z}\}$ can be recovered, up to a shift and reflection of $\mathbb{Z}$, in the case when the number of vertices with $\theta(z) \neq 0$ is finite:

Theorem 1.1. Suppose that attached to each site $z \in \mathbb{Z}$ is a coin with bias $\theta(z)$, and only finitely many of these coins have non-zero bias. Allow a simple random walker to generate observations by tossing, at each move, the coin attached to its current position. Then it is possible to determine the biases $\{\theta(z)\}_{z \in \mathbb{Z}}$, up to a shift and reflection of $\mathbb{Z}$, using only a single observation of the infinite sequence of these coin tosses and no information about the path of the random walker.

Another related model was introduced by Benjamini and Kesten [BK96] (and independently, by Den Hollander and Keane). Instead of attaching a coin with bias $\theta(z)$ to each $z \in \mathbb{Z}^{d}$, a fixed color is assigned to each vertex. The random walker reports only the sequence of colors visited, while just as in our generalization of the Harris-Keane model, the path itself is hidden. Further investigations of the model introduced in [BK96] can be found in [How96a, How96b, Lin99, LM02, LM03, Ma99, MR03a, MR03b] and a survey of results (up to 1998) can be found in [Kes98].

This paper is organized as follows. Theorem 1.1 holds more generally, where the observed random variables are not restricted to be coin tosses. Some definitions and the statement of the more general Theorem 2.3 can be found in Section 2. The main requirements of the proof of this theorem are a strong law of large numbers, and some algebraic results, found in Section 3. Because the special case of coin tosses is more readily understood, a separate proof of Theorem 1.1 is supplied, after which Theorem 2.3 is proven, both in Section 4. Finally, some unsolved problems are mentioned in Section 5. 


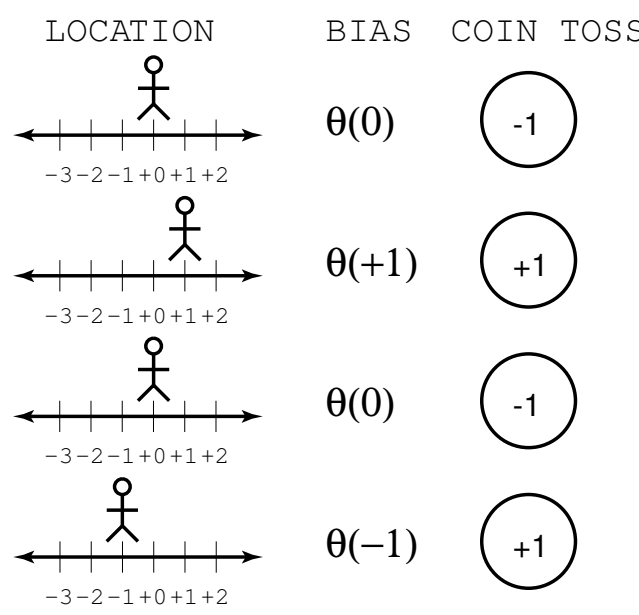

Figure 1: A walker performing his coin flips. We only see the right-hand column, but would like to recover the center column!

\section{Definitions and Statement of Result}

Let $q$ be a probability mass function on $\mathbb{Z}$, and $q^{* n}$ its $n$-fold convolution. Assume that

$$
\sum_{z \in \mathbb{Z}} z q(z)=0 \text { and } \sum_{z \in \mathbb{Z}} z^{2} q(z)<\infty .
$$

Also, we will assume that $q$ is symmetric:

$$
q(z)=q(-z) \text { for all } z \in \mathbb{Z} .
$$

In addition, to simplify the exposition we will suppose that

$$
\text { g.c.d. }\left\{n: q^{* n}(0)>0\right\}=1 \text {. }
$$

Remark. Condition (3) rules out, for example, $q=\frac{1}{2} \delta_{-1}+\frac{1}{2} \delta_{+1}$, the mass function corresponding to the increments of simple random walk on $\mathbb{Z}$. However, the results in this paper (in particular, Theorem 2.3) can be obtained for periodic random walks by considering the process observed at times $\{r n+k: n \in \mathbb{N}\}$, where $r$ is the period (the left-hand side of $(3))$.

The random walk with increments distributed according to $q$ will be denoted $S=$ $\left\{S_{n}\right\}_{n=0}^{\infty}$ :

$$
\mathbb{P}\left[S_{n+1}-S_{n}=z \mid S_{0}, \ldots, S_{n}\right]=q(z) .
$$

We use the notation $P^{n}(z, w) \stackrel{\text { def }}{=} \mathbb{P}\left[S_{n}=w \mid S_{0}=z\right]$ and $u_{n} \stackrel{\text { def }}{=} P^{n}(0,0)$. 
Definition 2.1. A stochastic scenery is a map $\eta: \mathbb{Z} \rightarrow \mathcal{P}(\mathbb{R})$, where $\mathcal{P}(\mathbb{R})$ is the set of all probability measures on $\mathbb{R}$.

We assume that there is a known reference distribution $\alpha \in \mathcal{P}(\mathbb{R})$ which appears everywhere but finitely many vertices: that is,

$$
\left|\eta^{-1}(\mathcal{P} \backslash\{\alpha\})\right|<\infty
$$

Definition 2.2. The sequence of observations induced by $S$ and $\eta$ is the stochastic process $X=\left\{X_{n}\right\}_{n=0}^{\infty}$, whose law conditioned on $S$ has independent marginal distributions given by $\mathbb{P}\left[X_{n} \in \cdot \mid S\right]=\eta\left(S_{n}\right)$.

Theorem 2.3. For a random walk $S$ with symmetric increment distribution $q$ satisfying (1) and (3), and stochastic scenery $\eta$ obeying (4), let $X=\left\{X_{n}\right\}_{n=0}^{\infty}$ be the observations induced by $S$ and $\eta$. Then using only the observations $\left\{X_{n}\right\}_{n=0}^{\infty}$, we can determine a scenery $\hat{\eta}$ so that

$$
\hat{\eta}=\eta \circ T \text { a.s. }
$$

where $T: \mathbb{Z} \rightarrow \mathbb{Z}$ is either a shift or a shift composed with a reflection, and may depend on $\eta$.

Remark. As noted by a referee, if we are given an infinite sequence of observations then we do not need to be given the reference distribution $\alpha$, as it can be a.s. reconstructed from the observations.

\section{A few ingredients}

In this section, we first state Proposition 3.2, which shows how the observations $\left\{X_{n}\right\}$ can be used to determine a family of parameters $\left\{p_{\boldsymbol{t}}\right\}$. We show in Proposition 3.7 that we can express a new family of parameters $\left\{Q_{\boldsymbol{d}}^{k}\right\}$ as functions of $\left\{p_{\boldsymbol{t}}\right\}$. These new parameters determines the scenery $\eta$, and hence the scenery can be recovered from the observations $\left\{X_{n}\right\}$. (This is the substance of the proofs of Theorem 1.1 and Theorem 2.3 in Section 4.) We conclude the present section with a proof of Proposition 3.2.

Definition 3.1. (i) For a vector $\boldsymbol{t} \in \mathbb{N}^{k}$ (where $k \in \mathbb{N}$ ), and a bounded and measurable $\varphi: \mathbb{R} \rightarrow \mathbb{R}$, define

$$
p_{\boldsymbol{t}}(\varphi) \stackrel{\text { def }}{=} \sum_{z \in \mathbb{Z}} \mathbb{E}\left[\varphi\left(X_{0}\right) \varphi\left(X_{t_{1}}\right) \varphi\left(X_{t_{1}+t_{2}}\right) \cdots \varphi\left(X_{t_{1}+\cdots+t_{k}}\right) \mid S_{0}=z\right] .
$$


(ii) For $r \in \mathbb{N}$, let $\boldsymbol{p}^{k}(\varphi)$ be the vector with components

$$
\left\{p_{\boldsymbol{t}}(\varphi): \boldsymbol{t} \in\{1, \ldots, r\}^{k}\right\}
$$

For measurable functions $\varphi$, we will use the supremum norm $\|\varphi\|_{\infty}$. When we know that all the measures $\eta(z)$ are supported on a common set $E$, we will take this norm to be the essential supremum over $E$. For example, when we are dealing with coin tosses only, $\|\varphi\|_{\infty} \leq 1$ means that $\varphi$ is bounded by 1 on $\{-1,1\}$, a condition satisfied by $\varphi(x)=x$.

Proposition 3.2. Fix a measurable function $\varphi$ with $\|\varphi\|_{\infty} \leq 1$ and $\int_{\mathbb{R}} \varphi d \alpha=0$, and a vector $\boldsymbol{t} \in \mathbb{N}^{s}$. Then there exists a sequence $\left\{g_{N}\right\}$ of measurable functions, $g_{N}: \mathbb{R}^{N} \rightarrow \mathbb{R}$, not depending on the stochastic scenery $\eta$, with

$$
\lim _{N \rightarrow \infty} g_{N}\left(X_{1}, \ldots, X_{N}\right)=p_{\boldsymbol{t}}(\varphi) \quad \text { almost surely. }
$$

The information in the collection $\left\{p_{\boldsymbol{t}}\right\}$ must be transformed into a more useful form for us to recover the stochastic scenery $\eta$. We provide now several results enabling this transformation.

Lemma 3.3. For a given transition semigroup $P^{t}$, and $m, r \in \mathbb{N}$, define the matrix $\boldsymbol{M}$ as

$$
\boldsymbol{M} \stackrel{\text { def }}{=}\left[\begin{array}{llll}
P^{1}(0,0) & P^{1}(0,1) & \cdots & P^{1}(0, m) \\
P^{2}(0,0) & P^{2}(0,1) & \cdots & P^{2}(0, m) \\
\vdots & \vdots & \ddots & \vdots \\
P^{r}(0,0) & P^{r}(0,1) & \cdots & P^{r}(0, m)
\end{array}\right] .
$$

Let $S$ be an aperiodic and symmetric random walk on $\mathbb{Z}$, whose increments have probability mass function $q$ : that is, $\mathbb{P}\left[S_{t+1}=z \mid S_{t}=w\right]=q(z-w)$.

(i) If $P^{t}$ is the transition semigroup for $S$, then $\boldsymbol{M}$ has rank $(m+1)$ for some $r$.

(ii) If $S$ is the simple random walk on $\mathbb{Z}$, and $P^{t}(0, z)=\mathbb{P}\left[S_{2 t}=2 z\right]$, then any $(m+1) \times$ $(m+1)$ square submatrix of $\boldsymbol{M}$ is non-singular.

Proof. If $\psi$ is the characteristic function of $q$, so that $\psi^{t}$ is the characteristic function of the $t$ th convolution power of $q$, then by Fourier inversion

$$
\frac{1}{2 \pi} \int_{-\pi}^{\pi} \cos (u j) \psi^{t}(u) d u=2 \mathbb{P}\left[S_{t}=j\right] .
$$


Suppose that for each $r$, the $m+1$ columns of $\boldsymbol{M}$ are linearly dependent, that is, there exist $\left\{a_{j}(r)\right\}_{j=0}^{m}$ (not all zero) so that

$$
\sum_{j=0}^{m} a_{j}(r) P^{t}(0, j)=0 \text { for all } t \leq r .
$$

By rescaling, it can be assumed that $\sum_{j=0}^{m} a_{j}(r)^{2}=1$. Thus there exist subsequential limits $\left\{a_{j}\right\}_{j=0}^{m}$ (not all zero) so that

$$
\sum_{j=0}^{m} a_{j} P^{t}(0, j)=0 \text { for all } t .
$$

Hence, the matrix $\boldsymbol{M}$ in (5) fails to have rank $(m+1)$ for any $r$ if and only if there exist $\left\{a_{j}\right\}_{j=0}^{m}$ satisfying (7). By (6), this is the same as

$$
\int_{-\pi}^{\pi} g(u) \psi^{t}(u) d u=0 \text { for all } t
$$

where $g(u)=\sum_{j=0}^{m} a_{j} \cos (u j)$. We now show that (8) cannot hold:

Observe that $g$ is an even function that is non-zero in a neighborhood of 0 , except possibly at 0 . It can be assumed without loss of generality (by multiplying the coefficients $a_{j}$ by -1 ) that $g$ is non-negative near 0 . Also $\psi$ is even, has value 1 at the origin, and is positive in a neighborhood $[-\delta, \delta]$ of 0 .

Choose $\delta_{1}<\delta$ so that

$$
B \stackrel{\text { def }}{=} \min _{|u| \leq \delta_{1}} \psi(u)>\max _{\delta<|u| \leq \pi} \psi(u) \stackrel{\text { def }}{=} A
$$

This is possible because, as $\psi$ is a characteristic function of an aperiodic distribution, $A<1$. Choose $\delta_{1}$ even smaller to ensure $\min _{\frac{\delta_{1}}{2} \leq|u| \leq \delta_{1}} g(u)=\epsilon>0$. Hence

$$
\begin{aligned}
\int_{-\pi}^{\pi} g(u) \psi^{t}(u) d u & =\int_{-\delta}^{\delta} g(u) \psi^{t}(u) d u+\int_{[-\pi, \pi] \backslash[-\delta, \delta]} g(u) \psi^{t}(u) d u \\
& \geq \int_{\delta_{1} / 2}^{\delta_{1}} g(u) \psi^{t}(u) d u+\int_{[-\pi, \pi] \backslash[-\delta, \delta]} g(u) \psi^{t}(u) d u \\
& \geq \frac{\delta_{1}}{2} \epsilon B^{t}-\|g\|_{\infty} 2 \pi A^{t}
\end{aligned}
$$


For $t$ large enough, the right-hand side of (9) is positive, contradicting (8). Thus the matrix (5) has rank $(m+1)$ when $r$ is sufficiently large, proving (i).

We now discuss the special case of simple random walk. Suppose there exist $a_{0}, \ldots, a_{m}$ and $t_{0}, t_{1}, \ldots, t_{m} \in 2 \mathbb{Z}$ so that for $t=t_{0}, \ldots, t_{m}$,

$$
\sum_{k=0}^{m} a_{k} \mathbb{P}\left[S_{2 t}=2 k\right]=0 .
$$

Then

$$
\sum_{k=0}^{m} a_{k} \frac{(2 t) !}{(t-k) !(t+k) !}=0
$$

Multiplying both sides of (10) by $\frac{(t+m) ! t !}{(2 t) !}$ yields

$$
0=\sum_{k=0}^{m} a_{k}(t+m) \cdots(t+k+1) t \cdots(t-k+1) .
$$

If $f_{k}(x) \stackrel{\text { def }}{=}(x+m) \cdots(x+k+1) x \cdots(x-k+1)$, then (11) shows that

$$
f(x) \stackrel{\text { def }}{=} \sum_{k=0}^{m} a_{k} f_{k}(x)=0 \text { for } x=t_{0}, \ldots, t_{m} .
$$

Since $f$ is a polynomial of degree $m$, and by (12) $f$ has $m+1$ zeros, it follows that $f \equiv 0$. But $f(-m)=0$ implies $a_{m}=0$.

Multiplying both side of (10) by $\frac{(t+m-1) ! t !}{(2 t) !}$ and arguing as above shows that $a_{m-1}=0$. Repeating this procedure shows that $a_{k}=0$ for all $k$, establishing (ii).

Remark 1. We have suppressed the dependence of the $r \times(m+1)$ matrix $\boldsymbol{M}$ on both $m$ and $r$. For each $m$, fix an $r_{m}$ so that $\boldsymbol{M}$ has rank $(m+1)$. In the sequel, the matrix $\boldsymbol{M}$ will depend only on $m$, having dimension $r_{m} \times(m+1)$. We will write $r$ for $r_{m}$.

We now introduce some notation:

Definition 3.4. For any function $\varphi: \mathbb{R} \rightarrow \mathbb{R}$, define

$$
\bar{\varphi}(z) \stackrel{\text { def }}{=} \int_{\mathbb{R}} \varphi d \eta(z), \quad \text { and } \quad \varphi_{0} \stackrel{\text { def }}{=} \varphi-\int_{\mathbb{R}} \varphi d \alpha .
$$

(Recall that $\eta(z) \in \mathcal{P}(\mathbb{R})$.) 
The parameters $\left\{\boldsymbol{Q}^{k}(\varphi)\right\}$ which will be used in the reconstruction of the scenery are now defined:

Definition 3.5. For $\varphi$ a bounded measurable function and $k \in \mathbb{N}$, define the vector $\boldsymbol{Q}^{k}(\varphi) \in \mathbb{R}^{[m]^{k}}$, indexed by $\boldsymbol{d} \in[m]^{k}$, by

$$
Q_{\boldsymbol{d}}^{k}(\varphi) \stackrel{\text { def }}{=} \sum_{\substack{\left(z_{1}, \ldots, z_{k+1}\right) \\\left|z_{j}-z_{j-1}\right|=d_{j}}} \bar{\varphi}\left(z_{1}\right) \bar{\varphi}\left(z_{2}\right) \cdots \bar{\varphi}\left(z_{k+1}\right) .
$$

(Here $[m] \stackrel{\text { def }}{=}\{0,1 \ldots, m\}$.)

Assumption (4) of Theorem 2.3 requires that the stochastic scenery $\eta$ differs from a reference distribution $\alpha$ at only finitely many vertices. With this in mind, we make the following definitions:

Definition 3.6. Let

$$
a \stackrel{\text { def }}{=} \inf \{j: \eta(j) \neq \alpha\}, \quad b \stackrel{\text { def }}{=} \sup \{j: \eta(j) \neq \alpha\}, \quad \text { and } \quad \ell \stackrel{\text { def }}{=} b-a .
$$

By assumption (4), $-\infty<a \leq b<\infty$.

Finally, we state the main algebraic result used in the proof of Theorem 2.3:

Proposition 3.7. If $m \geq \ell$ and $\int \varphi d \alpha=0$, then $\boldsymbol{Q}^{k}(\varphi) \in \mathbb{R}^{[m]^{k}}$ is a linear transformation of $\boldsymbol{p}^{k}(\varphi) \in \mathbb{R}^{r^{k}}$ (defined in Definition 3.1.)

Proof. Let us begin with the case of $k=1$. Let $\boldsymbol{M}$ be the matrix in (5), and $\boldsymbol{M}^{\prime}$ its left inverse, shown to exist in Lemma 3.3.

We have

$$
\begin{aligned}
p_{t}(\varphi) & =\sum_{z \in \mathbb{Z}} \mathbb{E}\left[\varphi\left(X_{t}\right) \varphi\left(X_{0}\right) \mid S_{0}=z\right] \\
& =\sum_{z \in \mathbb{Z}} \sum_{w \in \mathbb{Z}} \mathbb{E}\left[\varphi\left(X_{t}\right) \varphi\left(X_{0}\right) \mid S_{0}=z, S_{t}=w\right] \mathbb{P}\left[S_{t}=w \mid S_{0}=z\right] \\
& =\sum_{d \in \mathbb{Z}} P^{t}(0, d) \sum_{\substack{(z, w) \\
z-w=d}} \bar{\varphi}(z) \bar{\varphi}(w) .
\end{aligned}
$$

Because $q$ is symmetric, we have $P^{t}(0, d)=P^{t}(0,-d)$, and hence from (16) we conclude that

$$
p_{t}(\varphi)=\sum_{d=0}^{\infty} P^{t}(0, d) \sum_{\substack{(z, w) \\|z-w|=d}} \bar{\varphi}(z) \bar{\varphi}(w) .
$$


If $d \geq m$, then $d \geq \ell$ and the factor $\sum_{|z-w|=d} \bar{\varphi}(z) \bar{\varphi}(w)$ vanishes, so we have

$$
p_{t}(\varphi)=\sum_{d=0}^{m} M_{t, d} \cdot Q_{d}^{1}(\varphi) .
$$

Using Definition 3.1, we rewrite the collection of $r$ equations (17) as

$$
\boldsymbol{p}^{1}(\varphi)=\boldsymbol{M} \boldsymbol{Q}^{1}(\varphi)
$$

Multiplying both sides of (18) on the left by $\boldsymbol{M}^{\prime}$ yields $\boldsymbol{Q}^{1}(\varphi)=\boldsymbol{M}^{\prime} \boldsymbol{p}^{1}(\varphi)$.

Now consider the case $k=2$. We have

$$
\begin{aligned}
p_{\left(t_{1}, t_{2}\right)}(\varphi)= & \sum_{z, w, v \in \mathbb{Z}} \mathbb{E}\left[\varphi\left(X_{t_{1}+t_{2}}\right) \varphi\left(X_{t_{1}}\right) \varphi\left(X_{0}\right) \mid S_{0}=z, S_{t_{1}}=w, S_{t_{2}}=v\right] \\
& \times P^{t_{1}}(0, w-z) P^{t_{2}}(0, v-w) \\
= & \sum_{z, w, v \in \mathbb{Z}} \bar{\varphi}(z) \bar{\varphi}(w) \bar{\varphi}(v) P^{t_{1}}(0, w-z) P^{t_{2}}(0, v-w) \\
= & \sum_{d_{1}=-\infty}^{\infty} \sum_{d_{2}=-\infty}^{\infty} P^{t_{1}}\left(0, d_{1}\right) P^{t_{2}}\left(0, d_{2}\right) \sum_{\substack{(z, w, v) \\
w-z=d_{1}, v-w=d_{2}}} \bar{\varphi}(z) \bar{\varphi}(w) \bar{\varphi}(v) \\
= & \sum_{d_{1}=0}^{\infty} \sum_{d_{2}=0}^{\infty} P^{t_{1}}\left(0, d_{1}\right) P^{t_{2}}\left(0, d_{2}\right) \sum_{\substack{(z, w, v) \\
|w-z|=d_{1}, v-w \mid=d_{2}}} \bar{\varphi}(z) \bar{\varphi}(w) \bar{\varphi}(v) .
\end{aligned}
$$

Again, since $\sum_{|z-w|=d_{1},|w-v|=d_{2}} \bar{\varphi}(z) \bar{\varphi}(w) \bar{\varphi}(v)$ vanishes for $d_{1} \vee d_{2}>m \geq \ell$, we have

$$
p_{\left(t_{1}, t_{2}\right)}(\varphi)=\sum_{d_{1}=0}^{m} \sum_{d_{2}=0}^{m} P^{t_{1}}\left(0, d_{1}\right) P^{t_{2}}\left(0, d_{2}\right) \sum_{\begin{array}{c}
(z, w, v) \\
|z-w|=d_{1}, \\
|w-v|=d_{2}
\end{array}} \bar{\varphi}(z) \bar{\varphi}(w) \bar{\varphi}(v) .
$$

Recall that the tensor product of the matrix $\boldsymbol{M}$ in (5) with itself is given by

$$
\boldsymbol{M} \otimes \boldsymbol{M}=\left[\begin{array}{lll}
M_{1,0} \boldsymbol{M} & \cdots & M_{1, m} \boldsymbol{M} \\
\vdots & \ddots & \vdots \\
M_{r, 0} \boldsymbol{M} & \cdots & M_{r, m} \boldsymbol{M}
\end{array}\right]
$$

There are $r^{2}$ rows, indexed by $\boldsymbol{t} \in\left\{\left(t_{1}, t_{2}\right): 1 \leq t_{1}, t_{2} \leq r\right\}$, and $(m+1)^{2}$ columns indexed by $\boldsymbol{d} \in[m]^{2}=\left\{\left(d_{1}, d_{2}\right): d_{i}=0,1, \ldots, m\right\}$. Each row is of the form

$$
\left(P^{t_{1}}\left(0, d_{1}\right) P^{t_{2}}\left(0, d_{2}\right)\right)_{d_{1}, d_{2}=0}^{m} \text {. }
$$


Thus we can rewrite the equations (19) for $\boldsymbol{t} \in[r]^{2}$ compactly as

$$
\boldsymbol{p}^{2}(\varphi)=(\boldsymbol{M} \otimes \boldsymbol{M}) \boldsymbol{Q}^{2}(\varphi) .
$$

Because each $\boldsymbol{M}$ has rank $(m+1)$, the matrix $\boldsymbol{M} \otimes \boldsymbol{M}$ has rank $(m+1)^{2}$ (see [HJ91, Theorem 4.2.15]). Thus there is a left inverse $(\boldsymbol{M} \otimes \boldsymbol{M})^{\prime}$ to $\boldsymbol{M} \otimes \boldsymbol{M}$ and we can write

$$
\boldsymbol{Q}^{2}(\varphi)=(\boldsymbol{M} \otimes \boldsymbol{M})^{\prime} \boldsymbol{p}^{2}(\varphi) .
$$

In general, $\boldsymbol{M}^{k \otimes}$ has rank $(m+1)^{k}$, and hence has a left inverse, enabling us to write

$$
\boldsymbol{Q}^{k}(\varphi)=\left(\boldsymbol{M}^{k \otimes}\right)^{\prime} \boldsymbol{p}^{k}(\varphi) .
$$

Remark 2. The vectors $\boldsymbol{p}^{k}(\varphi)$ depend on the constant $r$ (see Definition 3.1), which we have taken to be the constant $r_{m}$ defined in Remark 1. Thus these vectors depend on $m$, although we have again suppressed this dependence in the notation.

Lemma 3.8. Let $\ell$ be the constant given in Definition 3.6. Then there exists a sequence of random variables $\left\{\ell_{n}\right\}_{n=0}^{\infty}$, not depending on $\eta$, with $\ell_{n}$ measurable with respect to $\sigma\left(X_{1}, \ldots, X_{n}\right)$, and so that $\ell_{n}=\ell$ eventually, a.s.

Recall that a class $\Phi$ of measurable functions on $\mathbb{R}$ is said to be measure determining if $\int_{\mathbb{R}} \varphi d \mu=\int_{\mathbb{R}} \varphi d \nu$ for each $\varphi \in \Phi$ implies $\mu=\nu$. Let $\Phi$ be a countable measure determining class of functions on $\mathbb{R}$ with $\|\varphi\|_{\infty} \leq 1$. We will enumerate $\Phi$ as $\left(\varphi_{1}, \varphi_{2}, \ldots\right)$. Also define $\Phi_{1} \stackrel{\text { def }}{=}\left\{\frac{f+c g}{\|f+c g\|_{\infty}}: f, g \in \Phi, c \in \mathbb{Q}\right\}$, and enumerate its elements as $\left(h^{1}, h^{2}, \ldots\right)$.

Lemma 3.9. Let $\mu$ and $\nu$ be two probability measures, neither equal to $\alpha$. Recall that $\psi_{0}=\psi-\int \psi d \alpha$. If $\mu \neq \nu$, there exists $\psi \in \Phi_{1}$ with

$$
\int \psi_{0} d \mu \neq 0, \quad \int \psi_{0} d \nu \neq 0, \quad \text { and } \quad \int \psi_{0} d \mu \neq \int \psi_{0} d \nu .
$$

Proof. Because $\mu \neq \nu$ and $\Phi$ is measure determining, there is an $f \in \Phi$ so that $\int f d \mu \neq$ $\int f d \nu$. By subtracting $\int f d \alpha$, we have $\int f_{0} d \mu \neq \int f_{0} d \nu$. Then one of $\int f_{0} d \mu$ and $\int f_{0} d \nu$ is nonzero; without loss of generality assume $\int f_{0} d \mu \neq 0$. Similarly, there is a $g \in \Phi$ so that $\int g d \nu \neq \int g d \alpha$, that is, $\int g_{0} d \nu \neq 0$.

Hence, for small enough $c \in \mathbb{Q}$, we have

$$
\int f_{0} d \mu+c \int g_{0} d \mu \neq 0, \text { and } \int f_{0} d \nu+c \int g_{0} d \nu \neq 0 .
$$


By taking $c$ even smaller, since $\int f_{0} d \mu \neq \int f_{0} d \nu$, we have

$$
\int f_{0} d \mu+c \int g_{0} d \mu \neq \int f_{0} d \nu+c \int g_{0} d \nu
$$

Thus, if $\psi=\frac{f+c g}{\|f+c g\|_{\infty}}$, so that $\psi_{0}=\frac{f_{0}+c g_{0}}{\|f+c g\|_{\infty}}$, we have from (22) and (23) that $\psi$ obeys (21).

We can now proceed with the proof of Lemma 3.8:

Proof of Lemma 3.8. As before, let $\boldsymbol{M}^{\prime}$ be the left inverse to the matrix $\boldsymbol{M}$ in (5).

Let $\ell^{h} \stackrel{\text { def }}{=} \sup \left\{|z-w|: \overline{h_{0}}(z) \overline{h_{0}}(w) \neq 0\right\}$. Recall that $\left(h^{1}, h^{2}, \ldots\right)$ is an enumeration of $\Phi_{1}$, and write $\ell^{j}$ for $\ell^{h^{j}}$. Lemma 3.9 guarantees that $\sup _{j \leq r} \ell^{j}=\ell$ for $r$ sufficiently large. Define

$$
T(m, h) \stackrel{\text { def }}{=} \pi_{m+1}\left(\boldsymbol{M}^{\prime} \boldsymbol{p}^{1}\left(h_{0}\right)\right),
$$

where $\pi_{m+1}$ is the projection onto the $m+1$ st coordinate.

Notice that

$$
T(m, h)= \begin{cases}\sum_{|z-w|=\ell^{h}} \overline{h_{0}}(z) \overline{h_{0}}(w) \neq 0 & \text { if } m=\ell^{h} \\ 0 & \text { if } m>\ell^{h}\end{cases}
$$

By Proposition 3.2, there exists for each $m$ and $j$ a sequence of random variables $\left\{T_{r}(m, j)\right\}_{r=1}^{\infty}$ so that almost surely,

$$
\lim _{r \rightarrow \infty} T_{r}(m, j)=T\left(m, h^{j}\right) .
$$

Let $\left\{s_{n}\right\}$ be any sequence with $\lim _{n \rightarrow \infty} s_{n}=\infty$. An examination of the proof of Proposition 3.2 shows that there are bounds on the variances of $T_{r}(m, j)$ uniform for $m \leq s_{n}$ and $j \leq s_{n}$. In particular, there is a sequence $f_{r}$ with $\lim _{r \rightarrow \infty} f_{r}=0$ so that

$$
\sup _{\substack{1 \leq j \leq s_{n} \\ \ell^{j}<m \leq s_{n}}} \mathbb{E}\left[T_{r}(m, j)^{2}\right] \leq f_{r} .
$$

We have

$$
\begin{aligned}
\mathbb{P}\left[\bigcup_{j=1}^{s_{n}} \bigcup_{m=\ell^{j}+1}^{s_{n}}\left\{T_{r}(m, j)>\frac{1}{n}\right\}\right] & \leq s_{n}^{2} n^{2} \sup _{\substack{1 \leq j \leq s_{n} \\
\ell^{j}<m \leq s_{n}}} \mathbb{E}\left[T_{r}(m, j)^{2}\right] \\
& \leq s_{n}^{2} n^{2} f_{r} .
\end{aligned}
$$


Let $\left\{B_{n}\right\}$ be a sequence so that $s_{n}^{2} n^{2} f_{B_{n}}$ is summable. By Borel-Cantelli, almost surely

$$
T_{B_{n}}(m, j) \leq \frac{1}{n} \quad \text { for } \ell^{j}<m \leq s_{n} \text { and } 1 \leq j \leq s_{n} \text {, eventually }
$$

Similarly, since $\lim _{r \rightarrow \infty} T_{r}\left(\ell^{j}, j\right)=T\left(\ell^{j}, j\right) \neq 0$, we can take a subsequence of $\left\{B_{n}\right\}$, which we will continue to denote by $\left\{B_{n}\right\}$, so that almost surely

$$
T_{B_{n}}\left(\ell^{j}, j\right)>\frac{1}{n} \quad \text { for } 1 \leq j \leq s_{n}, \text { eventually } .
$$

To summarize, the following holds almost surely: For $n$ sufficiently large, for all $j=$ $1, \ldots, s_{n}$

$$
T_{B_{n}}\left(\ell^{j}, j\right)>n^{-1} \text {, while } T_{B_{n}}(m, j) \leq n^{-1} \text { for } m=\ell^{j}+1, \ldots, s_{n} .
$$

Consequently,

$$
\sup \left\{m \leq s_{n}: T_{B_{n}}(m, j)>\frac{1}{n}\right\}=\ell^{j} \quad \text { for } 1 \leq j \leq s_{n}
$$

for $n$ sufficiently large. Finally,

$$
\sup _{j \leq s_{n}} \sup \left\{m \leq s_{n}: T_{B_{n}}(m, j)>\frac{1}{n}\right\}=\sup _{j \leq s_{n}} \ell^{j}=\ell
$$

for $n$ sufficiently large. [Since $\sup _{j \leq r} \ell^{j}=\ell$ for all but finitely many $r$.]

We still need a proof of Proposition 3.2; it is similar to the proof of [LPP01, Theorem 6.1]. Recall that $u_{n}=P^{n}(0,0)$. The only facts we need about $P^{n}$ are

$$
\sum_{n=0}^{\infty} u_{n}^{2}=\infty
$$

and

$$
\lim _{n \rightarrow \infty} \frac{P^{n-k}(z, w)}{P^{n-j}(u, v)}=1 \text { for all } u, v, z, w \in \mathbb{Z} \text { and } j, k \in \mathbb{N}
$$

That the transition probabilities satisfy (25) and (26) is easily seen from a local central limit theorem, for example [Woe00, Theorem 13.10]. 
Proof of Proposition 3.2. For

$$
w(m, n) \stackrel{\text { def }}{=} \sum_{k=m+1}^{n} u_{k}^{2} \quad \text { and } \quad Z_{k} \stackrel{\text { def }}{=} \varphi\left(X_{k}\right) \varphi\left(X_{k+t_{1}}\right) \cdots \varphi\left(X_{k+t_{1}+\cdots+t_{s}}\right),
$$

define the linear estimator

$$
L_{m, n} \stackrel{\text { def }}{=} \frac{1}{w(m, n)} \sum_{k=m+1}^{n} u_{k} Z_{k}
$$

Notice that $\mathbb{E}\left[L_{m, n}\right]=\frac{1}{w(m, n)} \sum_{k=m+1}^{n} u_{k}^{2} p_{\boldsymbol{t}, k}(\varphi)$, where we define

$$
p_{\boldsymbol{t}, k} \stackrel{\text { def }}{=} \sum_{z \in \mathbb{Z}} \mathbb{E}\left[Z_{k} \mid S_{k}=z\right] \frac{P^{k}(0, z)}{u_{k}} .
$$

Since $\lim _{k \rightarrow \infty} \frac{u_{k}}{P^{k}(0, z)}=1$ for all $z$, it follows that $\lim _{k \rightarrow \infty} p_{\boldsymbol{t}, k}(\varphi)=p_{\boldsymbol{t}}(\varphi)$ and

$$
\lim _{m \rightarrow \infty} \mathbb{E}\left[L_{m, n}\right]=p_{\boldsymbol{t}}(\varphi)
$$

The strategy of the proof is to find sequences $\left\{m_{i}\right\}$ and $\left\{n_{i}\right\}$ so that

$$
\lim _{N \rightarrow \infty} \frac{1}{N} \sum_{i=1}^{N} L_{m_{i}, n_{i}}=p_{\boldsymbol{t}}(\varphi) \text { almost surely } .
$$

We prove (29) by showing that $\operatorname{Cov}\left(Z_{j}, Z_{k}\right)$ is small for $|j-k|$ large, and consequently $\operatorname{Cov}\left(L_{m_{i}, n_{i}}, L_{m_{\tau}, n_{\tau}}\right)$ is small for $|i-\tau|$ large.

For $\boldsymbol{z} \in \mathbb{Z}^{s+1}$ and $j \in \mathbb{N}$, define the event

$$
H_{\boldsymbol{t}}(j, \boldsymbol{z}) \stackrel{\text { def }}{=}\left\{S_{j}=z_{0}, S_{j+t_{1}}=z_{1}, \ldots, S_{j+t_{1}+\cdots+t_{s}}=z_{s}\right\} .
$$

Then by conditioning on positions of $S$,

$$
\mathbb{E}\left[Z_{j} Z_{k}\right]=\sum_{\boldsymbol{z}, \boldsymbol{w} \in \mathbb{Z}^{s+1}} \mathbb{E}\left[Z_{j} Z_{k} \mid H_{\boldsymbol{t}}(j, \boldsymbol{z}) \cap H_{\boldsymbol{t}}(k, \boldsymbol{w})\right] \mathbb{P}\left[H_{\boldsymbol{t}}(j, \boldsymbol{z}) \cap H_{\boldsymbol{t}}(k, \boldsymbol{w})\right]
$$

Suppose that $k>j+t_{1}+\cdots+t_{s}$. Notice that

$$
\mathbb{E}\left[Z_{j} Z_{k} \mid H_{\boldsymbol{t}}(j, \boldsymbol{z}) \cap H_{\boldsymbol{t}}(k, \boldsymbol{w})\right]=\prod_{i=0}^{s} \bar{\varphi}\left(z_{i}\right) \prod_{j=0}^{s} \bar{\varphi}\left(w_{j}\right)
$$


and

$$
\begin{aligned}
\mathbb{P}\left[H_{\boldsymbol{t}}(j, \boldsymbol{z}) \cap H_{\boldsymbol{t}}(k, \boldsymbol{w})\right]= & P^{j}\left(0, z_{0}\right) \mathbb{P}\left[H_{\boldsymbol{t}}(j, \boldsymbol{z}) \mid S_{j}=z_{0}\right] \\
& \times P^{k-\left(j+t_{1}+\cdots+t_{s}\right)}\left(z_{s}, w_{0}\right) \mathbb{P}\left[H_{\boldsymbol{t}}(k, \boldsymbol{w}) \mid S_{k}=w_{0}\right] .
\end{aligned}
$$

Combining (31) and (32) shows that $\mathbb{E}\left[Z_{j} Z_{k}\right]$ is equal to

$$
\begin{aligned}
\sum_{\boldsymbol{z}, \boldsymbol{w} \in \mathbb{Z}^{s+1}}\left(\prod_{i=0}^{s} \bar{\varphi}\left(z_{i}\right) \prod_{j=0}^{s} \bar{\varphi}\left(w_{j}\right)\right) P^{j}\left(0, z_{0}\right) \mathbb{P}\left[H_{\boldsymbol{t}}(j, \boldsymbol{z}) \mid S_{j}=z_{0}\right] \\
\quad \times P^{k-\left(j+t_{1}+\cdots+t_{s}\right)}\left(z_{s}, w_{0}\right) \mathbb{P}\left[H_{\boldsymbol{t}}(k, \boldsymbol{w}) \mid S_{k}=w_{0}\right] .
\end{aligned}
$$

By similar reasoning, $\mathbb{E}\left[Z_{j}\right] \mathbb{E}\left[Z_{k}\right]$ is equal to

$$
\begin{aligned}
\sum_{\boldsymbol{z}, \boldsymbol{w} \in \mathbb{Z}^{s+1}}\left(\prod_{i=0}^{s} \bar{\varphi}\left(z_{i}\right) \prod_{j=0}^{s} \bar{\varphi}\left(w_{j}\right)\right) P^{j}\left(0, z_{0}\right) \mathbb{P}\left[H_{\boldsymbol{t}}(j, \boldsymbol{z}) \mid S_{j}=z_{0}\right] & \\
& \times P^{k}\left(0, w_{0}\right) \mathbb{P}\left[H_{\boldsymbol{t}}(k, \boldsymbol{w}) \mid S_{k}=w_{0}\right] .
\end{aligned}
$$

Because of the definitions of $a$ and $b$ in (15), if $D \geq|a| \vee|b|$, then for any $z \in \mathbb{Z}$ with $|z|>D$, we have $\bar{\varphi}(z)=0$.

Thus if $D \geq|a| \vee|b|$,

$$
\begin{aligned}
\left|\operatorname{Cov}\left(Z_{j}, Z_{k}\right)\right|=\left|\mathbb{E}\left[Z_{j} Z_{k}\right]-\mathbb{E}\left[Z_{j}\right] \mathbb{E}\left[Z_{k}\right]\right| \\
\leq \sum_{\substack{-D \leq z_{0} \leq D \\
\text {-D } z_{s} \leq D \\
-D \leq w_{0} \leq D}} P^{j}\left(0, z_{0}\right)\left|P^{k-\left(j+t_{1}+\cdots+t_{s}\right)}\left(z_{s}, w_{0}\right)-P^{k}\left(0, w_{0}\right)\right|
\end{aligned}
$$

since $\|\varphi\|_{\infty} \leq 1$.

For sequences $\left\{m_{i}\right\}_{i=1}^{\infty}$ and $\left\{n_{i}\right\}_{i=1}^{\infty}$ with $m_{i}<n_{i}$, define

$$
L_{i} \stackrel{\text { def }}{=} L_{m_{i}, n_{i}}=\frac{1}{w\left(m_{i}, n_{i}\right)} \sum_{j=m_{i}+1}^{n_{i}} u_{j} Z_{j} .
$$

For any sequence $\left\{\epsilon_{i}\right\}$, and integer $D$, we will inductively define sequences $\left\{m_{i}\right\}$ and $\left\{n_{i}\right\}$ so that for $\tau>i$,

$$
\left|\operatorname{Cov}\left(L_{i}, L_{\tau}\right)\right|<C \epsilon_{i}
$$


provided $D \geq|a| \vee|b|$. For now, assume that $D$ is indeed chosen so that $D \geq|a| \vee|b|$. To begin, let $m_{1}=n_{1}=1$. Now assume that the pair $\left(m_{i}, n_{i}\right)$ has already been defined. Pick $m_{i+1}$ large enough so that

if $j \leq n_{i}$ and $k>m_{i+1}$, then

$$
\left|P^{k-\left(j+t_{1}+\cdots+t_{s}\right)}(z, w)-P^{k}(0, w)\right| \leq C_{1}(D) \epsilon_{i} u_{k} \text { for all }-D \leq z, w \leq D .
$$

This is possible by (26).

Next, pick $n_{i+1}$ so that

$$
w\left(m_{i+1}, n_{i+1}\right) \geq w\left(m_{i+1}\right),
$$

where $w(m) \stackrel{\text { def }}{=} w(0, m)$. Combining (34) and (37) shows that

$$
\left|\operatorname{Cov}\left(Z_{j}, Z_{k}\right)\right| \leq(2 D)^{3} C_{1}(D) \epsilon_{i} u_{j} u_{k}=C_{2}(D) \epsilon_{i} u_{j} u_{k}
$$

for $j<n_{i}$ and $k>m_{i+1}$.

From the definition (35) and the bound (39) we have

$$
\begin{aligned}
\left|\operatorname{Cov}\left(L_{i}, L_{\tau}\right)\right| & =\left|\frac{1}{w\left(m_{i}, n_{i}\right)} \frac{1}{w\left(m_{\tau}, n_{\tau}\right)} \sum_{j=m_{i}+1}^{n_{i}} \sum_{k=m_{\tau}+1}^{n_{\tau}} u_{j} u_{k} \operatorname{Cov}\left(Z_{j}, Z_{k}\right)\right| \\
& \leq \frac{1}{w\left(m_{i}, n_{i}\right)} \frac{1}{w\left(m_{\tau}, n_{\tau}\right)} C_{2}(D) \epsilon_{i} \sum_{j=m_{i}+1}^{n_{i}} u_{j}^{2} \sum_{k=m_{\tau}+1}^{n_{\tau}} u_{k}^{2} \\
& =C_{2}(D) \epsilon_{i} .
\end{aligned}
$$

Next, we show that

$$
\sup _{i \geq 1} \mathbb{E}\left[L_{i}^{2}\right]<M<\infty
$$

Notice that

$$
\mathbb{E}\left[L_{i}^{2}\right] \leq \frac{1}{w\left(m_{i}, n_{i}\right)^{2}}\left\{2 \sum_{\substack{m_{i}<j \leq k \leq n_{i} \\|j-k| \leq t_{1}+\cdots+t_{s}}} u_{j} u_{k} \mathbb{E}\left[Z_{j} Z_{k}\right]+2 \sum_{\substack{m_{i}<j, k \leq n_{i} \\ j+t_{1}+\cdots+t_{s}<k}} u_{j} u_{k} \mathbb{E}\left[Z_{j} Z_{k}\right]\right\}
$$

By (26), for each fixed $k$, we have $\lim _{j \rightarrow \infty} \frac{u_{j}}{u_{j+k}}=1$, and hence $u_{j+k} \leq C_{3} u_{j}$ for $k=$ $0,1, \ldots, t_{1}+\cdots+t_{s}$. Consequently, since $\|\varphi\|_{\infty} \leq 1$ and hence $Z_{j} Z_{k} \leq 1$,

$$
\sum_{\substack{m_{i}<j \leq k \leq n_{i} \\|j-k| \leq t_{1}+\cdots+t_{s}}} u_{j} u_{k} \mathbb{E}\left[Z_{j} Z_{k}\right] \leq\left(t_{1}+\cdots+t_{s}\right) C_{3} \sum_{j=m_{i}+1}^{n_{i}} u_{j}^{2}=C_{4} w\left(m_{i}, n_{i}\right) .
$$


Since $\mathbb{E}\left[Z_{j} Z_{k}\right]$ is equal to (33), we have

$$
\mathbb{E}\left[Z_{j} Z_{k}\right] \leq \sum_{z_{0}=-D}^{D} P^{j}\left(0, z_{0}\right) \sum_{z_{s}=-D}^{D} \sum_{w_{0}=-D}^{D} P^{k-\left(j+t_{1}+\cdots+t_{s}\right)}\left(z_{s}, w_{0}\right) \leq C_{5}(D) u_{j} u_{k-j},
$$

again by (26). Thus,

$$
2 \sum_{\substack{m_{i}<j, k \leq n_{i} \\ j+t_{1}+\cdots+t_{s}<k}} u_{j} u_{k} \mathbb{E}\left[Z_{j} Z_{k}\right] \leq C_{6}(D) \sum_{j=m_{i}+1}^{n_{i}} u_{j}^{2} \sum_{k=j+1}^{n_{i}} u_{k} u_{k-j}
$$

Now applying the Cauchy-Schwarz inequality yields

$$
\sum_{k=j+1}^{n_{i}} u_{k} u_{k-j} \leq \sqrt{w\left(j, n_{i}\right) w\left(1, n_{i}-j\right)} \leq w\left(n_{i}\right)
$$

Plugging (44) into (43) yields

$$
2 \sum_{\substack{m_{i}<j, k \leq n_{i} \\ j+t_{1}+\cdots+t_{s}<k}} u_{j} u_{k} \mathbb{E}\left[Z_{j} Z_{k}\right] \leq C_{6}(D) w\left(n_{i}\right) w\left(m_{i}, n_{i}\right) .
$$

Then, using (45) and (42) in (41) yields

$$
\mathbb{E}\left[L_{i}^{2}\right] \leq \frac{C_{4}}{w\left(m_{i}, n_{i}\right)}+C_{6}(D) \frac{w\left(n_{i}\right)}{w\left(m_{i}, n_{i}\right)} .
$$

Now, since by $(38) w\left(m_{i}, n_{i}\right) \geq w\left(m_{i}\right)$, it follows that $w\left(n_{i}\right) \leq 2 w\left(m_{i}, n_{i}\right)$. Hence,

$$
\mathbb{E}\left[L_{i}^{2}\right] \leq \frac{C_{4}}{w\left(m_{i}, n_{i}\right)}+2 C_{6}(D) \leq M
$$

where $M$ doesn't depend on $i$.

If we choose $\epsilon_{i}=i^{-3}$, then a strong law for weakly correlated and centered random variables (see [Loè78, Theorem 37.7.A]) applied to the sequence $\left\{L_{i}-\mathbb{E}\left[L_{i}\right]\right\}_{i \geq 1}$ implies that $\frac{1}{N} \sum_{i=1}^{N}\left(L_{i}-\mathbb{E}\left[L_{i}\right]\right) \rightarrow 0$.

Thus, from (28), we have $\lim _{i \rightarrow \infty} \mathbb{E}\left[L_{i}\right]=p_{\boldsymbol{t}}(\varphi)$ and $\frac{1}{N} \sum_{i=1}^{N} L_{i} \rightarrow p_{\boldsymbol{t}}(\varphi)$.

To summarize, for each $D$ we get a sequence of functions $\left\{g_{N}^{D}\right\}_{N=1}^{\infty}$ so that

$$
\text { if } D \geq|a| \vee|b| \text {, then } g_{N}^{D}\left(X_{1}, \ldots, X_{N}\right) \stackrel{N \rightarrow \infty}{\longrightarrow} p_{\boldsymbol{t}}(\varphi) \text { a.s. }
$$


By a diagonalization argument, there is a sequence of integers $\left\{r_{n}\right\}$ so that for each $D$, the limit $A(D) \stackrel{\text { def }}{=} \lim _{n} g_{r_{n}}^{D}\left(X_{1}, \ldots, X_{r_{n}}\right)$ exists, and moreover for each $D$,

$$
\left|g_{r_{n}}^{D}\left(X_{1}, \ldots, X_{r_{n}}\right)-A(D)\right|<n^{-1} \text { for } n \geq D .
$$

From (46) it follows that $A(D)=p_{\boldsymbol{t}}(\varphi)$ for $D \geq|a| \vee|b|$, and consequently a.s.

$$
g_{r_{D}}^{D}\left(X_{1}, \ldots, X_{r_{D}}\right) \stackrel{D \rightarrow \infty}{\longrightarrow} p_{\boldsymbol{t}}(\varphi) .
$$

\section{Proofs of Theorem 1.1 and Theorem 2.3}

Here is an outline of the proof of Theorem 2.3: In the last section, it was shown in Proposition 3.2 that the parameters $\left\{p_{\boldsymbol{t}}\right\}$ can be recovered from the data $\left\{X_{1}, X_{2}, \ldots\right\}$. We transformed these parameters in Proposition 3.7 into the family $\left\{Q_{\boldsymbol{d}}\right\}$. Now we must show how to use this information to determine the laws $\{\eta(z)\}$.

Before we prove Theorem 2.3 in its full generality, we offer the proof of Theorem 1.1, the case where the observable random variables are coin tosses. The exposition is much clearer in this special case. The proof is given in its full generality later in this section.

Theorem 1.1. Let $S$ be a symmetric and aperiodic random walk, and label each $z \in \mathbb{Z}$ with a coin having bias $\theta(z)$. If only finitely many vertices have $\theta(z) \neq 0$, then there exist a sequence $\left\{\theta_{n}\right\}_{n=0}^{\infty}$ with $\theta_{n}$ measurable with respect to $\sigma\left(X_{1}, \ldots, X_{n}\right)$, and a shift and reflection $T$ (depending on $\eta$ ) so that

$$
\lim _{n \rightarrow \infty} \theta_{n}(z)=\theta \circ T(z) \text { for all } z \in \mathbb{Z}, \text { a.s. }
$$

Let us first introduce some notation.

Definition 4.1. Let $\left(x_{1}, x_{2}, \ldots, x_{n}\right)$ be a finite, ordered sequence. Define $\left[x_{1}, x_{2}, \ldots, x_{n}\right]$ as

$$
\left[x_{1}, x_{2}, \ldots, x_{n}\right] \stackrel{\text { def }}{=}\left\{\left(x_{1}, x_{2}, \ldots, x_{n}\right),\left(x_{n}, x_{n-1}, \ldots, x_{1}\right)\right\} .
$$

Proposition 4.2. Let $\theta: \mathbb{Z} \rightarrow[0,1]$ have compact support. Then, given $\ell=b-a$ as defined in (15),

$$
[\theta(a), \theta(a+1), \ldots, \theta(b-1), \theta(b)]
$$

can be uniquely determined from $\left\{\boldsymbol{p}^{k}(I)\right\}$, where $I$ is the identity map. 
Proof. We will write $Q_{\boldsymbol{d}}^{k}$ for $Q_{\boldsymbol{d}}^{k}(I)$, where $I$ is the identity map.

By Proposition 3.7 it is enough to construct (48) using only $\left\{\boldsymbol{Q}^{k}\right\}$.

Our method will be to first determine the biases at $a$ and $b$, and then work inwards. In the case where $k=1$ and $\boldsymbol{d}=(\ell)$, we have

$$
\frac{1}{2} Q_{\ell}^{1}=\frac{1}{2} \sum_{\substack{\left(z_{1}, z_{2}\right) \\\left|z_{2}-z_{1}\right|=\ell}} \theta\left(z_{1}\right) \theta\left(z_{2}\right)=\theta(a) \theta(b),
$$

as the only pairs $\left(z_{1}, z_{2}\right)$ with $\left|z_{2}-z_{1}\right|=\ell$ and with $\theta\left(z_{1}\right) \theta\left(z_{2}\right) \neq 0$ are $(a, b)$ and $(b, a)$.

Notice that

$$
Q_{(\ell, \ell)}^{2}=\sum_{\substack{\left(z_{1}, z_{2}, z_{3}\right) \\\left|z_{2}-z_{1}\right|=\ell,\left|z_{3}-z_{2}\right|=\ell}} \theta\left(z_{1}\right) \theta\left(z_{2}\right) \theta\left(z_{3}\right)=\theta(a) \theta(b)\{\theta(a)+\theta(b)\}
$$

and so $\theta(a)+\theta(b)=2 Q_{(\ell, \ell)}^{2} / Q_{\ell}^{1}$. We can conclude that

$$
\begin{aligned}
\{\theta(a), \theta(b)\} & =\left\{\frac{(\theta(a)+\theta(b)) \pm \sqrt{(\theta(a)+\theta(b))^{2}-4 \theta(a) \theta(b)}}{2}\right\} \\
& =\left\{Q_{(\ell, \ell)}^{2} / Q_{\ell}^{1} \pm \sqrt{\left(Q_{(\ell, \ell)}^{2} / Q_{\ell}^{1}\right)^{2}-Q_{\ell}^{1} / 2}\right\} .
\end{aligned}
$$

Now we move on to the unordered pair $\{\theta(a+1), \theta(b-1)\}$. Notice that

$$
Q_{(\ell, 1)}^{2}=\sum_{\substack{\left(z_{1}, z_{2}, z_{3}\right) \\\left|z_{2}-z_{1}\right|=\ell \\\left|z_{3}-z_{2}\right|=1}} \theta\left(z_{1}\right) \theta\left(z_{2}\right) \theta\left(z_{3}\right)=\theta(a) \theta(b)\{\theta(a+1)+\theta(b-1)\}
$$

and

$$
\begin{aligned}
Q_{(\ell, 1,1, \ell, 1)}^{5}= & \sum_{\substack{\left(z_{1}, z_{2}, z_{3}, z_{4}, z_{5}\right) \\
\left|z_{2}-z_{1}\right|=\ell,\left|z_{3}-z_{2}\right|=1 \\
\left|z_{4}-z_{3}\right|=1,\left|z_{5}-z_{4}\right|=\ell \\
\left|z_{6}-z_{5}\right|=1}} \theta\left(z_{1}\right) \theta\left(z_{2}\right) \theta\left(z_{3}\right) \theta\left(z_{4}\right) \theta\left(z_{5}\right) \theta\left(z_{6}\right) \\
= & 2\{\theta(a) \theta(b)\}^{2} \theta(a+1) \theta(b-1)
\end{aligned}
$$

Hence,

$$
\theta(a+1)+\theta(b-1)=2 Q_{(\ell, 1)}^{2} / Q_{\ell}^{1} \quad \text { and } \quad \theta(a+1) \theta(b-1)=2 Q_{(\ell, 1,1, \ell, 1)}^{5} /\left(Q_{\ell}^{1}\right)^{2}
$$


and so the unordered pair $\{\theta(a+1), \theta(b-1)\}$ can be written as a function of $\left(\boldsymbol{Q}^{1}, \ldots, \boldsymbol{Q}^{5}\right)$.

The cross-product $\theta(a) \theta(a+1)+\theta(b) \theta(b+1)$ allows us to write $[\theta(a), \theta(a+1), \theta(b-1), \theta(b)]$ as a function of the unordered pairs $\{\theta(a), \theta(b)\}$ and $\{\theta(a+1), \theta(b-1)\}$. We have

$$
\begin{aligned}
Q_{(\ell, \ell, 1)}^{3}= & \sum_{\substack{\left(z_{1}, z_{2}, z_{3}, z_{4}\right) \\
\left|z_{2}-z_{1}\right|=\ell,\left|z_{3}-z_{2}\right|=\ell,\left|z_{4}-z_{3}\right|=1}} \theta\left(z_{1}\right) \theta\left(z_{2}\right) \theta\left(z_{3}\right) \theta\left(z_{4}\right) \\
= & \theta(a) \theta(b)\{\theta(a) \theta(a+1)+\theta(b) \theta(b 11)\},
\end{aligned}
$$

and so

$$
\theta(a) \theta(a+1)+\theta(b) \theta(b-1)=2 Q_{(\ell, \ell, 1)}^{3} / Q_{\ell}^{1} .
$$

We conclude that $[\theta(a), \theta(a+1), \theta(b-1), \theta(b)]$ can be written as a function of the $\left\{\boldsymbol{Q}^{k}\right\}$.

Continuing in this way, we work down to the center to obtain (48), using only $\left\{\boldsymbol{Q}^{k}\right\}$.

Proof of Theorem 1.1. If $\ell$ is given, then by Proposition 4.2, it is clear that the theorem holds. That is, for each $\ell$, we can construct a scenery $\theta_{n}^{\ell}$ depending only on $\sigma\left(X_{1}, \ldots, X_{n}\right)$ measurable random variables, and so that $\theta_{n}^{\ell} \rightarrow \theta \circ T$. Then by Lemma 3.8, the sequence $\theta_{n}^{\ell_{n}}$ will satisfy the requirements of the Theorem.

We now provide a proof of our result in its full generality. Recall the notation given in Definition 3.4: As $\eta(z)$ is a probability measure on $\mathbb{R}$ for each $z \in \mathbb{Z}$,

$$
\bar{\varphi}(z)=\int_{\mathbb{R}} \varphi d \eta(z), \quad \text { and } \quad \varphi_{0}=\varphi-\int_{\mathbb{R}} \varphi d \alpha .
$$

Proof of Theorem 2.3. We will show how to use the collection $\mathcal{Q} \stackrel{\text { def }}{=}\left\{\boldsymbol{Q}^{k}(\varphi)\right\}_{\varphi \in \Phi_{1}, k \in \mathbb{N}}$ and $\ell$ to construct a stochastic scenery which is a shift and/or a reflection of $\eta$. The conclusion of the Theorem will then follow from applications of Proposition 3.2, Proposition 3.7, and Lemma 3.8 similarly to the proof of Theorem 1.1.

Fix a bounded and measurable $\varphi$. We have that

$$
\begin{aligned}
Q_{\ell}^{1}\left(\varphi_{0}\right) & =2 \overline{\varphi_{0}}(a) \overline{\varphi_{0}}(b) \\
Q_{(\ell, \ell)}^{2}\left(\varphi_{0}\right) & =\overline{\varphi_{0}}(a) \overline{\varphi_{0}}(b)\left\{\overline{\varphi_{0}}(a)+\overline{\varphi_{0}}(b)\right\} .
\end{aligned}
$$

Assume first that $\overline{\varphi_{0}}(a) \overline{\varphi_{0}}(b) \neq 0$. In this case, we can solve in (49) to obtain

$$
\overline{\varphi_{0}}(a) \overline{\varphi_{0}}(b)=Q_{\ell}^{1}\left(\varphi_{0}\right) / 2 \quad \text { and } \quad \overline{\varphi_{0}}(a)+\overline{\varphi_{0}}(b)=2 Q_{(\ell, \ell)}^{2}\left(\varphi_{0}\right) / Q_{\ell}^{1}\left(\varphi_{0}\right) \text {. }
$$


We use the identities in (50) to get

$$
\left\{\overline{\varphi_{0}}(a), \overline{\varphi_{0}}(b)\right\}=\left\{\frac{Q_{(\ell, \ell)}^{2}\left(\varphi_{0}\right)}{Q_{\ell}^{1}\left(\varphi_{0}\right)} \pm \sqrt{\left(\frac{Q_{(\ell, \ell)}^{2}\left(\varphi_{0}\right)}{Q_{\ell}^{1}\left(\varphi_{0}\right)}\right)^{2}-Q_{\ell}^{1}\left(\varphi_{0}\right) / 2}\right\}
$$

We proceed inductively: assume we have determined, for $k \leq \ell / 2$,

$$
\left\{\left(\overline{\varphi_{0}}(a), \ldots, \overline{\varphi_{0}}(a+k-1)\right),\left(\overline{\varphi_{0}}(b), \ldots, \overline{\varphi_{0}}(b-k+1)\right)\right\} .
$$

We have

$$
\begin{aligned}
Q_{(\ell, k, k, \ell)}^{4}\left(\varphi_{0}\right) & =\left(\overline{\varphi_{0}}(a) \overline{\varphi_{0}}(b)\right)^{2}\left\{\overline{\varphi_{0}}(a+k)+\overline{\varphi_{0}}(b-k)\right\}, \\
Q_{(\ell, k, k, \ell, k)}^{5}\left(\varphi_{0}\right) & =2\left(\overline{\varphi_{0}}(a) \overline{\varphi_{0}}(b)\right)^{2} \overline{\varphi_{0}}(a+k) \overline{\varphi_{0}}(b-k) .
\end{aligned}
$$

By assumption, $\overline{\varphi_{0}}(a) \overline{\varphi_{0}}(b) \neq 0$, so we can solve in (53) (using (50)) to get

$$
\overline{\varphi_{0}}(a+k) \overline{\varphi_{0}}(b-k)=\frac{2 Q_{(\ell, k, k, \ell, k)}^{5}}{\left(Q_{\ell}^{1}\left(\varphi_{0}\right)\right)^{2}} \quad \text { and } \quad \overline{\varphi_{0}}(a+k)+\overline{\varphi_{0}}(b-k)=\frac{4 Q_{(\ell, k, k, \ell)}^{4}\left(\varphi_{0}\right)}{\left(Q_{\ell}^{1}\left(\varphi_{0}\right)\right)^{2}} \text {. }
$$

Thus, similarly to (51), we can determine

$$
\left\{\overline{\varphi_{0}}(a+k), \overline{\varphi_{0}}(b-k)\right\}
$$

as a function of elements of $\mathcal{Q}$.

Now define $m \stackrel{\text { def }}{=} \sup \left\{j \leq k-1: \overline{\varphi_{0}}(a+j) \neq \overline{\varphi_{0}}(b-j)\right\}$. If there is no such $j$, there is no problem in finding

$$
\left\{\left(\overline{\varphi_{0}}(a), \ldots, \overline{\varphi_{0}}(a+k)\right),\left(\overline{\varphi_{0}}(b), \ldots, \overline{\varphi_{0}}(b-k)\right)\right\}
$$

from (52) and (54), so without loss of generality, assume such a $j$ exists.

Now notice that

$$
Q_{(\ell, m, m, \ell, k, k, \ell)}^{7}=\left(\overline{\varphi_{0}}(a) \overline{\varphi_{0}}(b)\right)^{3}\left\{\overline{\varphi_{0}}(a+m) \overline{\varphi_{0}}(b-k)+\overline{\varphi_{0}}(b-m) \overline{\varphi_{0}}(a+k)\right\}
$$

and hence

$$
\overline{\varphi_{0}}(a+m) \overline{\varphi_{0}}(b-k)+\overline{\varphi_{0}}(b-m) \overline{\varphi_{0}}(a+k)=\frac{8 Q_{(\ell, m, m, \ell, k, k, \ell)}^{7}}{\left(Q_{\ell}^{1}\left(\varphi_{0}\right)\right)^{3}}
$$


From the two unordered pairs (which we already know)

$$
\left\{\overline{\varphi_{0}}(a+m), \overline{\varphi_{0}}(b-m)\right\} \quad \text { and } \quad\left\{\overline{\varphi_{0}}(a+k), \overline{\varphi_{0}}(b-k)\right\}
$$

we can get two possible pairings:

$$
\begin{aligned}
& F_{\text {good }} \stackrel{\text { def }}{=}\left\{\left(\overline{\varphi_{0}}(a+m), \overline{\varphi_{0}}(a+k)\right),\left(\overline{\varphi_{0}}(b-m), \overline{\varphi_{0}}(b-k)\right)\right\} \\
& F_{b a d} \stackrel{\text { def }}{=}\left\{\left(\overline{\varphi_{0}}(a+m), \overline{\varphi_{0}}(b-k)\right),\left(\overline{\varphi_{0}}(b-m), \overline{\varphi_{0}}(a+k)\right)\right\} .
\end{aligned}
$$

From these two pairings we can compute the values

$$
\begin{gathered}
f_{\text {good }} \stackrel{\stackrel{\text { def }}{=} \overline{\varphi_{0}}(a+m) \overline{\varphi_{0}}(a+k)+\overline{\varphi_{0}}(b-m) \overline{\varphi_{0}}(b-k)}{\quad \text { and }} \\
f_{\text {bad }} \stackrel{\text { def }}{=} \overline{\varphi_{0}}(a+m) \overline{\varphi_{0}}(b-k)+\overline{\varphi_{0}}(b-m) \overline{\varphi_{0}}(a+k)
\end{gathered}
$$

Since we have written $f_{\text {bad }}$ in (56) as a function of elements of $\mathcal{Q}$, we can use these elements to discard the pairing $F_{b a d}$ in (57) from which it comes. If $f_{b a d}=f_{\text {good }}$, we cannot determine the proper pairing. But this happens if and only if

$$
\overline{\varphi_{0}}(a+k)\left(\overline{\varphi_{0}}(a+m)-\overline{\varphi_{0}}(b-m)\right)=\overline{\varphi_{0}}(b-k)\left(\overline{\varphi_{0}}(a+m)-\overline{\varphi_{0}}(b-m)\right) .
$$

Since $m$ was defined so that $\overline{\varphi_{0}}(a+m)-\overline{\varphi_{0}}(b-m) \neq 0$, we conclude that (59) is valid if and only if $\overline{\varphi_{0}}(a+k)=\overline{\varphi_{0}}(b-k)$. But in this case, it is clear that (55) can be found from (52) and (54).

Otherwise, we can pick out $F_{\text {good }}$ from (57), and hence we can determine (55).

Thus, by induction, we can determine

$$
\{(\bar{\varphi}(a), \ldots, \bar{\varphi}(b)),(\bar{\varphi}(b), \ldots, \bar{\varphi}(a))\} \text {. }
$$

To summarize, we have just shown for any bounded measurable function $\varphi$ obeying the condition $\overline{\varphi_{0}}(a) \overline{\varphi_{0}}(b) \neq 0$ we can determine

$$
[\bar{\varphi}(a), \ldots, \bar{\varphi}(b)]
$$

Now suppose that $\overline{\varphi_{0}}(a) \overline{\varphi_{0}}(b)=0$. Recall that the elements of $\Phi_{1}$ are enumerated as $\left(h_{1}, \ldots, h_{n}, \ldots\right)$. If we define $n^{*}$ as the smallest $n$ so that $\left\{\overline{h_{n}}(a)-\int_{\mathbb{R}} h_{n} d \alpha, \overline{h_{n}}(b)-\int_{\mathbb{R}} h_{n} d \alpha\right\}$ has distinct non-zero elements, then Lemma 3.9 guarantees that $n^{*}<\infty$. Let $\psi \stackrel{\text { def }}{=} h_{n^{*}}$.

There is a $c \in \mathbb{Q}$ small enough so that

$$
\overline{\varphi_{0}+c \psi_{0}}(a) \neq 0 \text { and } \overline{\varphi_{0}+c \psi_{0}}(b) \neq 0 .
$$


We can determine $\left[\overline{c \psi_{0}}(a), \ldots, \overline{c \psi_{0}}(b)\right]$, and $\left[\overline{\varphi_{0}+c \psi_{0}}(a), \ldots, \overline{\varphi_{0}+c \psi_{0}}(b)\right]$.

Thus we can determine the two quantities

$$
\begin{aligned}
& {\left[\overline{\varphi_{0}+c \psi_{0}}(a)-\overline{c \psi_{0}}(a), \ldots, \overline{\varphi_{0}+c \psi_{0}}(b)-\overline{c \psi_{0}}(b)\right]} \\
& {\left[\overline{\varphi_{0}+c \psi_{0}}(a)-\overline{c \psi_{0}}(b), \ldots, \overline{\varphi_{0}+c \psi_{0}}(b)-\overline{c \psi_{0}}(a)\right]}
\end{aligned}
$$

But the end points of the second expression are nonzero for $c$ small enough, while at least one endpoint in the first express is zero. Thus we can pick out the first expression, which allows us to determine (61).

The careful reader will have noticed that our use of Lemma 3.9 requires that $\eta(a) \neq \eta(b)$. If this is not the case, then $\overline{\varphi_{0}}(a)=\overline{\varphi_{0}}(b)=0$. We can then apply the entire procedure with $\ell-2, a+1, b-1$ replacing $\ell, a$ and $b$ respectively.

Now we have shown that we can determine

$$
\left(\left[\overline{\varphi_{1}}(a), \ldots, \overline{\varphi_{1}}(b)\right],\left[\overline{\varphi_{2}}(a), \ldots, \overline{\varphi_{2}}(b)\right], \ldots\right) .
$$

Our goal now is to find

$$
\left[\left(\overline{\varphi_{k}}(a)\right)_{k=1}^{\infty},\left(\overline{\varphi_{k}}(a+1)\right)_{k=1}^{\infty}, \ldots,\left(\overline{\varphi_{k}}(b)\right)_{k=1}^{\infty}\right],
$$

which, since $\Phi$ is measure determining, yields $[\eta(a), \eta(a+1), \ldots, \eta(b)]$. Again, we use induction. Suppose that we know

$$
\left[\left(\overline{\varphi_{1}}(a), \ldots, \overline{\varphi_{k-1}}(a)\right), \ldots,\left(\overline{\varphi_{1}}(b), \ldots, \overline{\varphi_{k-1}}(b)\right)\right] .
$$

From (62), we have in particular

$$
\left[\overline{\varphi_{k}}(a), \ldots, \overline{\varphi_{k}}(b)\right] .
$$

There are two possible extensions of (63) using (64):

$$
\begin{aligned}
G_{\text {good }} & \stackrel{\text { def }}{=}\left[\left(\overline{\varphi_{1}}(a), \ldots, \overline{\varphi_{k-1}}(a), \overline{\varphi_{k}}(a)\right), \ldots,\left(\overline{\varphi_{1}}(b), \ldots, \overline{\varphi_{k-1}}(b), \overline{\varphi_{k}}(b)\right)\right] \\
G_{b a d} & \stackrel{\text { def }}{=}\left[\left(\overline{\varphi_{1}}(a), \ldots, \overline{\varphi_{k-1}}(a), \overline{\varphi_{k}}(b)\right), \ldots,\left(\overline{\varphi_{1}}(b), \ldots, \overline{\varphi_{k-1}}(b), \overline{\varphi_{k}}(a)\right)\right]
\end{aligned}
$$

We want to guarantee that we can pick out $G_{\text {good }}$ from $\left\{G_{\text {good }}, G_{b a d}\right\}$ given in (65).

Suppose there is some $j$ so that

$$
\left(\overline{\varphi_{1}}(a+j), \ldots, \overline{\varphi_{k-1}}(a+j)\right) \neq\left(\overline{\varphi_{1}}(b-j), \ldots, \overline{\varphi_{k-1}}(b-j)\right) .
$$


This is without loss of generality, since if there is no such $j$, then of course we can extend (63) to obtain

$$
\left[\left(\overline{\varphi_{1}}(a), \ldots, \overline{\varphi_{k}}(a)\right), \ldots,\left(\overline{\varphi_{1}}(b), \ldots, \overline{\varphi_{k}}(b)\right)\right] .
$$

Since the linear functionals on $\mathbb{R}^{k-1}$ separate points, there exist $\boldsymbol{c} \in \mathbb{R}^{k-1}$ so that if $T_{c}(\boldsymbol{x})=$ $\langle\boldsymbol{c}, \boldsymbol{x}\rangle$, then

$$
T_{c}\left(\left(\overline{\varphi_{1}}(a+j), \ldots, \overline{\varphi_{k-1}}(a+j)\right)\right) \neq T_{c}\left(\left(\overline{\varphi_{1}}(b-j), \ldots, \overline{\varphi_{k-1}}(b-j)\right)\right) .
$$

Since for any function $\varphi$ we can recover (60), by linearity of expectation, for the function $T_{c}\left(\left(\varphi_{1}, \ldots, \varphi_{k-1}\right)\right)+\varphi_{k}$ we can determine

$$
\left.\left[T_{c}\left(\overline{\varphi_{1}}(a), \ldots, \overline{\varphi_{k-1}}(a)\right)+\overline{\varphi_{k}}(a), \ldots, T_{c}\left(\overline{\varphi_{1}}(b), \ldots, \overline{\varphi_{k-1}}(b)\right)+\overline{\varphi_{k}}(b)\right)\right]
$$

But from (65), we can determine

$$
\begin{aligned}
& {\left[T_{c}\left(\overline{\varphi_{1}}(a), \ldots, \overline{\varphi_{k-1}}(a)\right)+\overline{\varphi_{k}}(a), \ldots, T_{c}\left(\overline{\varphi_{1}}(b), \ldots, \overline{\varphi_{k-1}}(b)\right)+\overline{\varphi_{k}}(b)\right]} \\
& {\left[T_{c}\left(\overline{\varphi_{1}}(a), \ldots, \overline{\varphi_{k-1}}(a)\right)+\overline{\varphi_{k}}(b), \ldots, T_{c}\left(\overline{\varphi_{1}}(b), \ldots, \overline{\varphi_{k-1}}(b)\right)+\overline{\varphi_{k}}(a)\right]}
\end{aligned}
$$

It thus suffices to show that these two sets cannot be the same. If the sets in (69) are the same, then either $\overline{\varphi_{k}}(a+j)=\overline{\varphi_{k}}(b-j)$ for all $j$, in which case there is no problem in obtaining (67), or

$$
T_{c}\left(\overline{\varphi_{1}}(a+j), \ldots, \overline{\varphi_{k-1}}(a+j)\right)=T_{c}\left(\overline{\varphi_{1}}(b-j), \ldots, \overline{\varphi_{k-1}}(b-j)\right) \text { for all } j .
$$

But $T_{c}$ was defined to rule out this possibility. Hence we can distinguish the "correct" extension in (65), and thus we can determine (67).

By induction, we can then determine

$$
\left[\left(\overline{\varphi_{k}}(a)\right)_{k=1}^{\infty}, \ldots,\left(\overline{\varphi_{k}}(b)\right)_{k=1}^{\infty}\right]
$$

Because the $\left\{\varphi_{k}\right\}$ are measure determining, from (70) we can determine

$$
[\eta(a), \ldots, \eta(b)]
$$

Pick one $\ell$-tuple from $[\eta(a), \ldots, \eta(b)]$, and call it $\left(\eta_{0}(1), \ldots, \eta_{0}(\ell)\right)$. Finally, define $\hat{\eta}$ by

$$
\hat{\eta}(z)=\left\{\begin{array}{ll}
\alpha & \text { if } z<1 \text { or } z>\ell \\
\eta_{0}(z) & \text { if } 1 \leq z \leq \ell
\end{array} .\right.
$$




\section{Unsolved Problems}

There remain a couple of questions still requiring resolution:

1. It follows from results in [Lin99] that if we allow infinitely many biased coins, then there exist bias configurations $\{\theta(z): z \in \mathbb{Z}\}$ that cannot be recovered up to a reflection and shift. We have restricted attention to finitely many biased coins; however, the methods of this paper do allow recovery of infinitely many biases (up to reflection and shift) if the biased coins are sufficiently sparse. It remains an open question to determine for which infinite sets of integers $S$ can one recover the biases of biased coins located in $S$ from the tosses observed by a random walker.

2. We have shown in Theorem 2.3 that for symmetric walks with finite variance increments, we can recover finitely many unknown distributions up to a shift and/or a reflection. We have not been able to generalize the algebraic results in Section 3 to walks which are asymmetric, yet still mean zero. In particular, Lemma 3.3 needs to be proven for a matrix with entries $\left\{P^{i}(0, j): 1 \leq i \leq r,-m \leq j \leq m\right\}$. We expect the following to hold:

Conjecture 1. In the setting of theorem 2.3, suppose that the symmetry assumption on the walk $S$ is replaced by the assumption that the walk $S$ has mean-zero, nonsymmetric increments of finite variance. Then $\eta$ can be recovered up to a shift only.

3. In Theorem 2.3, is it possible to replace the symmetric random walk on $\mathbb{Z}$ satisfying (1), by a general null-recurrent Markov chain that satisfies $\sum_{n} u_{n}^{2}=\infty$ ?

(Here $u_{n}$ is the probability that the chain returns to its starting state after $n$ steps.) The goal is to recover $\eta$ up to an automorphism of the Markov chain.

Acknowledgements. We are grateful to Rich Bass for useful discussions, and to Alan Hammond and Gabor Pete for helpful comments on the manuscript.

\section{References}

[BK96] I. Benjamini and H. Kesten. Distinguishing sceneries by observing the scenery along a random walk path. J. Anal. Math., 69:97-135, 1996.

[HJ91] R.A. Horn and C.R. Johnson. Topics in matrix analysis. Cambridge University Press, Cambridge, 1991. 
[HK97] M. Harris and M. Keane. Random coin tossing. Probab. Th. Rel. Fields, 109:2737, 1997.

[How96a] C.D. Howard. Detecting defects in periodic scenery by random walks on Z. Rand. Struc. Algorithms, 8(1):59-74, 1996.

[How96b] C.D. Howard. Orthogonality of measures induced by random walks with scenery. Combin. Probab. Comput., 5:247-256, 1996.

[Kes98] H. Kesten. Distinguishing and reconstructing sceneries from observations along random walk paths. In D. Aldous and J. Propp, editors, Microsurveys in Discrete Probability, volume 41 of DIMACS Series in Discrete Mathematics and Theoretical Computer Science. American Mathematical Society, 1998.

[Lin99] E. Lindenstrauss. Indistinguishable sceneries. Rand. Struc. Algorithms, 14(1):7186, 1999.

[Loè78] M. Loève. Probability Theory II. Springer-Verlag, New York, NY, fourth edition, 1978.

[LM02] M. Löwe and H. Matzinger. Scenery reconstruction in two dimensions with many colors. Ann. Appl. Probab. 12(4): 1322-1347, 2002.

[LM03] M. Löwe and H. Matzinger. Reconstruction of sceneries with correlated colors. Stochastic Process. Appl. 105(2): 175-210, 2003.

[LPP01] D. Levin, R. Pemantle, and Y. Peres. A phase transition in random coin tossing. Ann. Probab., 29(4), 2001.

[Ma99] H. Matzinger. Reconstructing a three-color scenery by observing it along a simple random walk path. Rand. Struc. Algorithms 15(2): 196-207, 1999.

[MR03a] H. Matzinger and S. Rolles. Reconstructing a piece of scenery with polynomially many observations. Stochastic Process. Appl. 107(2): 289-300, 2003.

[MR03b] H. Matzinger and S. Rolles. Reconstructing a random scenery observed with random errors along a random walk path. Probab. Theory Related Fields 125(4): 539-577, 2003.

[Woe00] W. Woess. Random Walks on Infinite Graphs and Groups, volume 138 of Cambridge Tracts in Mathematics. Cambridge University Press, Cambridge, 2000. 